Philosophie ANTIQUE

\section{Philosophie antique}

Problèmes, Renaissances, Usages

$20 \mid 2020$

Nouvelles figures de Socrate

\title{
Pierre Aubenque
}

in memoriam (1929-2020)

\section{Jean-Baptiste Gourinat}

\section{CpenEdition}

\section{Journals}

Édition électronique

URL : https://journals.openedition.org/philosant/3562

DOI : 10.4000/philosant.3562

ISSN : 2648-2789

Éditeur

Éditions Vrin

\section{Édition imprimée}

Date de publication : 31 octobre 2020

Pagination : 5-6

ISBN : 978-2-7116-2977-0

ISSN : $1634-4561$

Référence électronique

Jean-Baptiste Gourinat, « Pierre Aubenque », Philosophie antique [En ligne], 20 | 2020, mis en ligne le 25 octobre 2020, consulté le 02 décembre 2022. URL : http://journals.openedition.org/philosant/3562 ; DOI : https://doi.org/10.4000/philosant.3562

\section{(c) (i) (3)}

Creative Commons - Attribution - Pas d'Utilisation Commerciale - Pas de Modification 4.0 International - CC BY-NC-ND 4.0

https://creativecommons.org/licenses/by-nc-nd/4.0/ 


\section{PIERRE AUBENQUE \\ IN MEMORIAM (1929-2020)}

Pierre Aubenque est mort le 23 février 2020, à l'âge de 90 ans. Né en juillet 1929 à l' Isle-Jourdain dans le Gers, il était un spécialiste internationalement reconnu d'Aristote, professeur de philosophie antique à l'université Paris-Sorbonne de 1970 à 1991, où il a dirigé le centre Léon Robin de 1973 à 1990, ainsi que l'UFR de Philosophie.

Surtout connu pour ses monographies sur Aristote, Le Problème de l'être chez Aristote (PUF, 1962), et La Prudence chez Aristote (PUF, 1963), il a aussi co-écrit un recueil de textes sur Sénèque avec Jean-Marie André (Sénéque, présentation, choix de textes, bibliographie, Seghers, 1964). Il a publié, seul ou en collaboration, de nombreux volumes collectifs, comme les actes du Symposium Aristotelicum de Cerisy-la-Salle en 1972, Études sur la Métaphysique d'Aristote (paru en 1979 chez Vrin), et les actes des nombreux séminaires du centre Léon Robin : Concepts et catégories dans la pensée antique (Vrin, 1980), Études sur Parménide, avec Denis O’ Brien et Jean Frère (Vrin, 1987), Études sur le Sophiste de Platon, éditées par M. Narcy (Bibliopolis, 1991), Aristote politique : études sur la Politique d'Aristote, publiées par A. Tordesillas (PUF, 1993). Il a également publié chez Beauchesne, en 1972, le débat de Davos entre Heidegger et Ernst Cassirer, ouvrage qui fut malheureusement retiré de la vente et pilonné sur demande des ayants droit de Heidegger. Il a également participé au volume des Stoïciens de La Pléiade (NRF, 1962), achevé sous la supervision de P.-M. Schuhl après le décès d'Émile Bréhier : il y a révisé la traduction du livre II de la Nature des dieux de Cicéron et celle du Traité du destin, ainsi que celle des Entretiens d'Épictète. Son intérêt pour le néoplatonisme est marqué entre autres par sa contribution importante au volume issu du colloque de Royaumont sur le néoplatonisme : « Plotin et le dépassement de l'ontologie grecque classique $\gg$, dans Le Néoplatonisme. Actes du colloque de Royaumont, 9-13 juin 1969, Éd. du CNRS, 1971. Deux recueils de ses articles ont été publiés chez Vrin, Problèmes aristotéliciens I. Philosophie théorique (2009) et Problèmes aristotéliciens II. Philosophie pratique (2011), et il a également publié en 2009 un essai intitulé Faut-il déconstruire la métaphysique? (PUF), issu de ses conférences de la chaire Étienne Gilson en 1997-1998, suivies à l'époque par un public considérable. Deux volumes d'hommage lui ont été

Philosophie antique, n²0 (2020), 5-7 
dédiés, Herméneutique et ontologie, publié sous la direction de R. Brague et J.-F. Courtine (PUF, 1990) et Ontologie et Dialogue, réuni par N.-L. Cordero (Vrin, 2000) à l'occasion de ses soixante-dix ans.

Exceptionnel connaisseur de la philosophie d'Aristote, il était aussi excellent germaniste que connaisseur des langues anciennes, et il avait une connaissance approfondie de la philosophie allemande moderne et contemporaine, en particulier de la pensée de Heidegger qu'il connut en Allemagne dans l'après-guerre, ce dont Jean Beaufret s'étonna le jour où il crut les faire se rencontrer pour la première fois. Il fut profondément marqué par la pensée de Heidegger, dont Le Problème de l'être, en posant le problème de l'unité de la métaphysique, porte la trace. Il ouvrit ainsi la voie en France à de nombreuses études d'histoire de la philosophie marquées par les questions de l'onto-théologie ou des thématiques heideggériennes, comme les travaux de Barbara Cassin et Michel Narcy ou de Rémi Brague, mais aussi de Jean-Luc Marion ou Jean-François Courtine. Mais il était également attentif aux questions posées par la logique, la philosophie analytique (il cite souvent Quine) et la linguistique, et par ses contemporains, comme Michel Foucault ou Jacques Derrida (qu'il ne croisa pas à l'École Normale Supérieure, contrairement au premier). On se souviendra par exemple qu'il a discuté à plusieurs reprises les thèses d'Émile Benveniste sur l'interprétation grammaticale des catégories d'Aristote ou que dans son livre Faut-il déconstruire la métaphysique? au titre derridien, un chapitre entier est consacré à Derrida.

Il a marqué des générations d'étudiants et de collègues par la force spéculative et la clarté exceptionnelle de sa pensée. Ses travaux sur Aristote, qui ont pris à bras-le-corps le problème de l'unité de la métaphysique d'Aristote, défendu sa structure dialectique et aporétique et parallèlement se sont attachés au statut de la philosophie pratique, ont profondément renouvelé l'histoire de la philosophie antique. Avec son contemporain Jacques Brunschwig, qu'il connut dès 1948 à l'École Normale Supérieure, il a longtemps dominé les études aristotéliciennes françaises. De la lecture de son œuvre résultent sans doute deux impressions dominantes qui peuvent paraître antithétiques, celle d'une pensée extrêmement claire, voire lumineuse, et celle d'une pensée aporétique - elles sont les deux faces d'une pensée rigoureuse et lucide. Comme il l'écrivait dans la préface de Faut-il déconstruire la métaphysique?, « on pourra s'étonner de l'atmosphère d'aporicité, d'absence de solutions, qui se détache de ces études, ou, en termes vulgaires, du pessimisme qu'elles semblent distiller ». Tous les participants du séminaire du centre Léon Robin, qu'il a animé pendant vingt ans, en prenant la suite de P.-M. Schuhl, se souviennent de l'art qu'il avait de résumer et de synthétiser immédiatement avec une grande exactitude chaque communication présentée, et d'en tirer sur-le-champ quelques questions incisives. Tous ses anciens étudiants se souviennent de la précision méticuleuse de ses cours, qui laissaient place 
à l'aporie et au doute, mais certainement pas à l'obscurité, et qui les munissaient de structures et de questions gravées dans leur mémoire.

Fils d'un instituteur, en 1947, à 18 ans à peine, il est reçu premier à l'École Normale Supérieure, où il restera imperméable à l'influence de Louis Althusser. Reçu premier à l'agrégation de philosophie trois ans plus tard, il fut ensuite pensionnaire de la fondation Thiers, avant d'occuper plusieurs postes successifs de maître-assistant à Montpellier puis de professeur à Besançon, Aix-Marseille et Paris-IV. Il enseigna aussi à Hambourg, et, après sa retraite française, à Leipzig. Sa thèse fut dirigée par Maurice de Gandillac, professeur de philosophie médiévale à la Sorbonne, mais il bénéficia aussi, selon ses propres termes dans la préface du Problème de l'être, des conseils de P.-M. Schuhl, d'Aimé Forest à Montpellier et de l'helléniste Paul Mazon à la Fondation Thiers. Outre ses responsabilités à la Sorbonne, il fut le directeur de la revue Les Études philosophiques et le secrétaire général de l'Institut international de philosophie. Il se retira à un âge assez précoce, au tout début de la soixantaine.

Membre du comité scientifique de Philosophie Antique depuis sa création en 2001, il y avait publié en 2003 un article, l'un de ses derniers, intitulé «Sens et fonction de l'aporie socratique ${ }^{1}$.

Jean-Baptiste GourinAT notice.

1. Je remercie Michel Gourinat et Michel Narcy pour leur aide dans la rédaction de cette 
